\title{
Covid-19: Remdesivir is helpful but not a wonder drug, say researchers
}

\author{
Elisabeth Mahase
}

The BMJ

Antiviral drug remdesivir helps patients hospitalised with covid-19 recover faster and may also reduce mortality, according to the US National Institutes of Health (NIH).

The drug-developed by US pharmaceutical company Gilead-is being tested through a randomised, controlled trial involving 1063 patients from several countries including the US, UK, and Singapore. The study began in February.

Currently, no official results have been published, however the $\mathrm{NIH}$ said that the independent data and safety monitoring board overseeing the trial met on 27 April to review data and shared their interim analysis with the study team.

They reported that the preliminary data suggests remdesivir was better than placebo in terms of time to recovery, the primary endpoint defined as being well enough for hospital discharge or returning to normal activity level.

The team carrying out the research in the UK expressed "cautious optimism" over the findings but stressed that other drugs would also be needed.

In its announcement, the NIH said, "Preliminary results indicate that patients who received remdesivir had a $31 \%$ faster time to recovery than those who received placebo $(\mathrm{p}<0.001)$. Specifically, the median time to recovery was 11 days for patients treated with remdesivir compared with 15 days for those who received placebo.

"Results also suggested a survival benefit, with a mortality rate of $8.0 \%$ for the group receiving remdesivir versus $11.6 \%$ for the placebo group ( $\mathrm{P}=0.059)$."

Remdesivir-which is given as an intravenous drug-has previously been tested against Ebola and two coronaviruses-Middle East respiratory syndrome and severe acute respiratory syndrome.

Speaking at a press briefing on 30 April, infectious disease consultant Brian Angus—-part of the UK team—said, "As far as the results are concerned, it's cautious optimism. There is some effect but it is not a wonder effect. We have to find out when is the best time to give this drug, who benefits more. There is still a lot of data to come out of this trial."

The researchers added that while only 46 of the patients included in the trial were from the UK, more could have been recruited if there had not been such a limited supply of the drug.
Max Parmar, director of the Medical Research Council Clinical Trials Unit at University College London, also stressed that other drugs will be needed in the fight against covid-19.

He said, "Most major diseases need combination treatment to show real progress, to attack the disease in a number of different ways. We see that in HIV, tuberculosis, and many cancers. Covid-19 is likely to be the same. If these results hold up over the next month or so, it suggests remdesivir may be one of the backbones of combination treatment, but we are going to need other drugs as well."

The team said the second stage of the trial will see patients given remdesivir in combination with another agent, likely to be a janus kinase inhibitor.

The early findings from this study come as another remdesivir trial, this time in China, reported in the Lancet. ${ }^{1}$ The paper said the study was forced to stop early because of difficulty recruiting covid-19 patients as the country brought the outbreak under control. A total of 237 patients aged over 18 from 10 hospitals in Wuhan were included.

The researchers concluded that "remdesivir was not associated with statistically significant clinical benefits." They reported adverse events in 102 (66\%) of 155 remdesivir recipients $v 50$ (64\%) of 78 placebo recipients.

Speaking on the current evidence to support remdesivir, Robin Ferner, honorary professor of clinical pharmacology at the University of Birmingham, told The BMJ, "In a pandemic it is desirable to have treatments assessed quickly, but what you need is reliable information.

"Expedited reviews or expedited publication are fine, but hinting that results are going to be positive only benefits the drug companies. It does not benefit the clinician. I, for example, would still not know if remdesivir was the right thing to give my patients."

Wang Y, Zhang D, Du G, et al. Remdesivir in adults with severe covid-19: a randomised, double-blind, placebo-controlled, multicentre trial. Lancet, 2020. www.thelancet.com/ journals/lancet/article/PIIS0140-6736(20)31022-9/fulltext.

Published by the BMJ Publishing Group Limited. For permission to use (where not already granted under a licence) please go to http://group.bmj.com/group/rights-licensing/ permissions 